\title{
Phenotypic Diversity of Non-heading Chinese Cabbage Germplasm Resources in Zhejiang Province
}

Haijiao Hu, Jinglei Wang, Tianhua Hu, Wuhong Wang, Qingzhen Wei, Chonglai Bao $₫$

Institute of Vegetables, Zhejiang Academy of Agricultural Sciences, Hangzhou, 310021, China

D Corresponding email: baocl@mail.zaas.ac.cn

International Journal of Horticulture, 2020, Vol.10, No.3 doi: 10.5376/ijh.2020.10.0003

Received: 04 Aug., 2020

Accepted: 17 Aug., 2020

Published: 31 Aug., 2020

Copyright $\odot 2020 \mathrm{Hu}$ et al., This article was first published in Molecular Plant Breeding in Chinese, and here was authorized to translate and publish the paper in English under the terms of Creative Commons Attribution License, which permits unrestricted use, distribution, and reproduction in any medium, provided the original work is properly cited.

Preferred citation for this article:

Hu H.J., Wang J.L., Hu T.H., Wang W.H., Wei Q.Z., and Bao C.L., 2020, Phenotypic diversity of non-heading Chinese cabbage germplasm resources in Zhejiang Province, International Journal of Horticulture, 10(3): 1-8 (doi: $\underline{10.5376 / \text { ijh.2020.10.0003) }}$

\begin{abstract}
In this study, 48 non-heading Chinese cabbage germplasms collected in the Third National Survey and Collection Action on Crop Germplasm Resources in Zhejiang Province were used as materials for phenotypic identification and genetic diversity analysis. The results showed that the variation coefficient of 10 quantitative characters ranged from $23.33 \%$ to $50.29 \%$, and the genetic diversity index ranged from 1.26 to 2.01 . And the variation coefficients of 9 quality traits ranged from $14.57 \%$ to $65.77 \%$, and the genetic diversity index ranged from 0.29 to 1.61 . There was a high correlation between different characters, and a significant positive correlation between plant height, plant width, leaf length and petiole length, besides, the number of lotus leaves was negatively correlated with other characters. The cumulative contribution rate of first 5 principal components is $76.587 \%$, which contains most of the information. The tested materials were classified into 5 groups by cluster analysis. The first group is Wuta-tsai, the second group is mainly erect-type leafy cabbage, the third group is Pak-choi, the fourth group is mainly milk Chinese cabbage and the fifth group is a hybrid group. This study provides theoretical basis for efficient utilization and breeding of non-heading Chinese cabbage germplasm resources in Zhejiang Province.
\end{abstract}

Keywords Non-heading Chinese cabbage; Germplasm; Phenotypic diversity; Correlation analysis; Principal component analysis

Non-heading Chinese cabbage (Brassica campestris ssp., Chinensis Makino) is one of the Brassica campestris varieties of Brassica genus in the family of Brassicaceae. It is native to southern China and is also one of the main leaf vegetables in China (Zhang, 2011, China Agricultural Science and Technology Press, pp.979). There are six varieties of Chinensis Makino, var. communis Tsen et Lee, var. rosularis Tsen et Lee, var. tsai-tai Hort, var. Taitsai Hort, var. Muticeps Hort, and var. Utilis Tsen et Lee (Feng et al., 2019). Common cabbage, also known as "Qingcai", "Qinggengcai", "Xiaoyoucai", "Xiaobaicai" in Chinese, is the most widely distributed, especially in the middle and lower reaches of the Yangtze River, accounting for 30\% to $40 \%$ of the total area of vegetable multiple planting (Hou and Song, 2012).

Zhejiang is a coastal province in southeast China, with complex and diverse terrain, including plain, basin, hill, mountain and island, mainly are hill and mountain, with obvious stereoscopic weather, so it has strong biodiversity (Wang, 1988). Local varieties have high adaptability to local climate conditions and are rich in types, which are the original materials for breed selection. Since the launch of the Third National Survey and Collection Action on Crop Germplasm Resources in Zhejiang Province, a total of 48 local non-heading Chinese cabbage varieties that have been continuously planted in different areas of Zhejiang have been collected. In order to systematically evaluate the diversity and understand the types and characteristics of local non-heading Chinese cabbage varieties in Zhejiang province, we conducted phenotypic evaluation and diversity analysis on 19 agronomic traits of 48 local cabbage varieties in 2019. This study will provide reference for the exploration of excellent agronomic traits and efficient application in breeding of non-heading Chinese cabbage germplasm resources in Zhejiang Province.

\section{Results and Analysis}

\subsection{Distribution of germplasm resources}

From June 2017 to June 2019, germplasm resources investigation group of Vegetable Institute, Zhejiang Academy of Agricultural Sciences collected 48 non-heading Chinese cabbage germplasm resources through systematic 
investigation in Zhejiang Province (Table 1), which were distributed in 10 prefecture-level cities and 23 counties and administrative regions (cities, districts, counties) in Zhejiang Province. Among them, 9 in Jiaxing, 8 in Quzhou, 7 in Hangzhou, 5 in Huzhou, 5 in Lishui, 4 in Zhoushan, 4 in Shaoxing, 3 in Ningbo, 2 in Jinhua, and 1 in Taizhou. In terms of counties, Tongxiang County, Jiaxing city have the most, a total of 8.

Table 1 Forty-eight collected non-heading Chinese cabbage germplasm resources

\begin{tabular}{|c|c|c|c|c|}
\hline Ordinal & Code & Varieties name & Collection data & Origin \\
\hline 1 & 2017332064 & Non-heading Chinese cabbage & 20170831 & Hangzhou Jiande \\
\hline 2 & 2017333083 & Savoying pak-choi & 20170919 & Ningbo Ninghai \\
\hline 3 & 2017334089 & Zhongjiaobai & 20170929 & Ningbo Fenghua \\
\hline 4 & 2018331414 & Non-heading Chinese cabbage & 20180831 & Jiaxing Tongxiang \\
\hline 5 & 2018331433 & Changgeng Non-heading Chinese cabbage & 20180831 & Jiaxing Tongxiang \\
\hline 6 & 2018331435 & Changgeng Non-heading Chinese cabbage & 20180831 & Jiaxing Tongxiang \\
\hline 7 & 2018332060 & Laobaicai & 20180615 & Lishui Jingning \\
\hline 8 & 2018332083 & Zisong & 20180615 & Lishui Jingning \\
\hline 9 & 2018332235 & Non-heading Chinese cabbage & 20180829 & Lishui Qingyuan \\
\hline 10 & 2018332414 & Yuangeng Non-heading Chinese cabbage & 20180829 & Quzhou Kaihua \\
\hline 11 & 2018332425 & Non-heading Chinese cabbage & 20180829 & Quzhou Kaihua \\
\hline 12 & 2018332459 & Black Non-heading Chinese cabbage & 20180829 & Quzhou Kaihua \\
\hline 13 & 2018333252 & Non-heading Chinese cabbage & 20180816 & Quzhou Qujiang \\
\hline 14 & 2018334168 & Youdonger & 20181121 & Shaoxing Zhuji \\
\hline 15 & 2018335022 & Siyueqing & 20180820 & Zhoushan Dinghai \\
\hline 16 & p330127053 & Lao Savoying pak-choi & 20180806 & Hangzhou Chunan \\
\hline 17 & p330127054 & Lao Non-heading Chinese cabbage & 20180806 & Hangzhou Chunan \\
\hline 18 & p330127069 & Dongdongqing & 20170922 & Hangzhou Chunan \\
\hline 19 & p330226003 & Zhong Non-heading Chinese cabbage & 20171113 & Ningbo Ninghai \\
\hline 20 & p330683026 & Youdonger & 20180612 & Shaoxing Shengzhou \\
\hline 21 & p330683035 & Gaojiaobai & 20180620 & Shaoxing Shengzhou \\
\hline 22 & p330781019 & Lanxi Gaojiaobai & 20180702 & Jinhua Lanxi \\
\hline 23 & p330783003 & Non-heading Chinese cabbage & 20170606 & Jinhua Dongyang \\
\hline 24 & 2018331442 & Changgeng Non-heading Chinese cabbage & 20180831 & Jiaxing Tongxiang \\
\hline 25 & p330111055 & Changgeng Non-heading Chinese cabbage & 20180530 & Hangzhou Fuyang \\
\hline 26 & p330127091 & Sanyueqing & 20180806 & Hangzhou Chunan \\
\hline 27 & p330424016 & Black Non-heading Chinese cabbage & 20180620 & Jiaxing Haiyan \\
\hline 28 & p330822007 & Gaogan Non-heading Chinese cabbage & 20180115 & Qushan Changshan \\
\hline 29 & p330822020 & Changshan Savoying pak-choi & 20180619 & Qushan Changshan \\
\hline 30 & p330483024 & Changgeng Non-heading Chinese cabbage & 20180801 & Jiaxing Tongxiang \\
\hline 31 & 2018331282 & Zhouwencai & 20180930 & Huzhou Changxing \\
\hline 32 & 2018331283 & Longxucai & 20180930 & Huzhou Changxing \\
\hline 33 & 2018331272 & Siyueqing & 20180930 & Huzhou Changxing \\
\hline 34 & 2018331273 & Aijiaobai & 20180930 & Huzhou Changxing \\
\hline 35 & 2018331445 & Changgeng Non-heading Chinese cabbage & 20181026 & Jiaxing Tongxiang \\
\hline 36 & 2018331464 & Aijiao Non-heading Chinese cabbage & 20181026 & Jiaxing Tongxiang \\
\hline 37 & 2018335006 & Heiyoutong & 20180925 & Zhoushan \\
\hline 38 & p331125014 & Shenzhuang Non-heading Chinese cabbage & 20181130 & Lishui Yunhe \\
\hline 39 & p330824025 & Savoying pak-choi & 20181122 & Quzhou Kaihua \\
\hline 40 & 2018334183 & Pak-choi rape & 20181113 & Shaoxing Zhuji \\
\hline 41 & 2018332415 & Can Non-heading Chinese cabbage & 20180829 & Quzhou Kaihua \\
\hline 42 & 2018334475 & Yangshetou Non-heading Chinese cabbage & 20181130 & Hangzhou Linan \\
\hline 43 & 2018332079 & Xiaobaye & 20180615 & Lishui Jingning \\
\hline 44 & 2019335015 & Eryueqing & 20190106 & Zhoushan Shengsi \\
\hline 45 & 2019335013 & Siyueman & 20190106 & Zhoushan Shengsi \\
\hline 46 & 2019333672 & Non-heading Chinese cabbage & 20190612 & Taizhou Huangyan \\
\hline 47 & p330502014 & Xiuhuajin & 20190614 & Huzhou Wuxing \\
\hline 48 & 2018331449 & Chaozhoucai & 20190618 & Jiaxing Tongxiang \\
\hline
\end{tabular}




\subsection{Genetic diversity analysis}

The genetic diversity analysis of 19 traits of 48 non-heading Chinese cabbage germplasm resources showed that there were large differences among different materials, and the variation of each trait was abundant (Table 2). The variation coefficients of the 10 quantitative traits ranged from $23.33 \%$ to $50.29 \%$, among which the rosette leaves number (RLN) and petiole length (PL) were the largest (more than 50\%). The minimum is crown diameter (CD). From big to small sort of $\mathrm{PL}(50.29 \%)>\mathrm{RLN}(50.15 \%)>\mathrm{SH}(39.83 \%)>\mathrm{PH}(39.79 \%)>\mathrm{PT}(37.30 \%)>\mathrm{LL}(32.32 \%)>\mathrm{PW}$ $(29.85 \%)>\mathrm{LW}(26.98 \%)>\mathrm{SH}(25.01 \%)>\mathrm{CD}(23.33 \%)$. The genetic diversity index of 10 quantitative traits ranged from 1.26 to 2.01 , in which the stem height ( $\mathrm{SH}$ ) was the highest and the RLN was the lowest.

Table 2 Variation and genetic diversity analysis of 10 quantitative traits of non-heading Chinese cabbage

\begin{tabular}{llllllll}
\hline Characters & Min. & Max. & Mean & $S D$ & Variance & $C V(\%)$ & $H$ \\
\hline Plant height, PH & 10.70 & 61.20 & 29.13 & 11.59 & 134.34 & 39.79 & 1.88 \\
Crown diameter, CD & 22.70 & 68.00 & 43.25 & 10.09 & 101.82 & 23.33 & 1.99 \\
Rosette leaves number, RLN & 6.00 & 54.00 & 13.29 & 6.67 & 44.42 & 50.15 & 1.26 \\
Leaf length, LL & 14.10 & 67.60 & 36.33 & 11.74 & 137.90 & 32.32 & 1.93 \\
Leaf width, LW & 5.80 & 33.70 & 15.67 & 4.23 & 17.88 & 26.98 & 1.79 \\
Petiole length, PL & 3.00 & 36.90 & 14.40 & 7.24 & 52.44 & 50.29 & 1.92 \\
Petiole width, PW & 1.50 & 5.50 & 3.35 & 1.00 & 1.00 & 29.85 & 1.98 \\
Petiole thick, PT & 0.40 & 1.40 & 0.74 & 0.28 & 0.08 & 37.30 & 1.82 \\
Stem height, SH & 1.00 & 5.90 & 3.19 & 1.27 & 1.61 & 39.83 & 2.01 \\
Stem width, SW & 1.30 & 4.80 & 2.39 & 0.60 & 0.36 & 25.01 & 1.83
\end{tabular}

The variation coefficients of 9 quality traits ranged from $14.57 \%$ to $65.77 \%$, among which, leaf shape (LS) was the largest and leaf type (LT) was the smallest (Table 3). From big to small sort of LT(65.77\%)>LM(61.49\%)>LSS $(57.17 \%)>\operatorname{PC}(48.17 \%)>\operatorname{LTS}(36.64 \%)>\operatorname{LC}(25.99 \%)>\operatorname{PT}(25.09 \%)>\operatorname{HS}(22.91 \%)>\operatorname{LS}(14.57 \%) . \quad$ The $\quad$ genetic diversity index of nine quality traits ranged from 0.29 to 1.61, with leaf shape being the highest and leaf type the lowest.

Table 3 Variation and genetic diversity analysis of 9 qualitative traits of non-heading Chinese cabbage.

\begin{tabular}{llllllll}
\hline Characters & Min. & Max. & Mean & $S D$ & Variance & $C V(\%)$ & $H$ \\
\hline Plant type, PT & 1 & 5 & 4.06 & 1.02 & 1.04 & 25.09 & 1.20 \\
Hourglass sign, HS & 1 & 2 & 1.79 & 0.41 & 0.17 & 22.91 & 0.51 \\
Leaf type, LT & 1 & 2 & 1.92 & 0.28 & 0.08 & 14.57 & 0.29 \\
Leaf shape, LS & 1 & 8 & 3.27 & 2.15 & 4.63 & 65.77 & 1.61 \\
Leaf tip shape, LTS & 1 & 4 & 2.92 & 1.07 & 1.14 & 36.64 & 1.31 \\
Leaf margin, LM & 1 & 4 & 1.42 & 0.87 & 0.76 & 61.49 & 0.78 \\
Leaf surface smoothness, LSS & 1 & 4 & 1.67 & 0.95 & 0.91 & 57.17 & 1.07 \\
Leaf color, LC & 2 & 7 & 3.67 & 0.95 & 0.91 & 25.99 & 1.27 \\
Petiole color, PC & 1 & 4 & 2.31 & 1.11 & 1.24 & & 48.17 \\
\hline
\end{tabular}

\subsection{Correlation analysis of quantitative characters}

Correlation analysis was conducted on 10 quantitative traits of non-heading Chinese cabbage resources, and the results showed that there were significant correlations among several traits (Table 4). Plant height, crown diameter, leaf length and petiole length were positively correlated with each other. Leaf width was significantly positively correlated with plant height, crown diameter and petiole thick, but significantly positively correlated with leaf length and petiole width. Rosette leaves number was negatively correlated with other traits, and was significantly different from leaf width, plant height, crown diameter and leaf length. Petiole width was significantly positively correlated with petiole thick and stem height, and positively correlated with leaf width and stem width. 
Table 4 The correlation coefficient between the quantitative traits of non-heading Chinese cabbage.

\begin{tabular}{|c|c|c|c|c|c|c|c|c|c|c|}
\hline Traits & $\mathrm{PH}$ & $\mathrm{CD}$ & RLN & LL & LW & PL & PW & PT & $\mathrm{SH}$ & SW \\
\hline $\mathrm{PH}$ & 1.00 & & & & & & & & & \\
\hline $\mathrm{CD}$ & $0.83^{* *}$ & 1.00 & & & & & & & & \\
\hline RLN & $-0.31^{*}$ & $-0.33^{*}$ & 1.00 & & & & & & & \\
\hline LL & $0.91^{* *}$ & $0.88^{* *}$ & $-0.33^{*}$ & 1.00 & & & & & & \\
\hline LW & $0.37^{* *}$ & $0.48^{* *}$ & $-0.52^{* *}$ & $0.30^{*}$ & 1.00 & & & & & \\
\hline PL & $0.72^{* *}$ & $0.59^{* *}$ & -0.08 & $0.77^{* *}$ & 0.04 & 1.00 & & & & \\
\hline PW & 0.08 & 0.11 & -0.14 & -0.08 & $0.29^{*}$ & 0.08 & 1.00 & & & \\
\hline PT & -0.09 & 0.03 & -0.20 & -0.22 & $0.40^{* *}$ & -0.23 & $0.67^{* *}$ & 1.00 & & \\
\hline $\mathrm{SH}$ & -0.11 & -0.06 & -0.03 & -0.21 & 0.15 & $-0.32^{*}$ & $0.41^{* *}$ & $0.36^{*}$ & 1.00 & \\
\hline SW & 0.10 & 0.15 & -0.20 & -0.06 & $0.32^{*}$ & $-0.31^{*}$ & $0.52^{*}$ & 0.21 & $0.58^{* *}$ & 1.00 \\
\hline
\end{tabular}

\subsection{Principal component analysis}

Principal component analysis was conducted on 19 traits of 48 non-heading Chinese cabbage germplasm resources, and the results showed that the cumulative contribution rate of the first five principal components was $76.587 \%$, containing most of the information of 19 traits (Table 5). Among them, the numerical value of the first principal component was 5.454, and the contribution rate was 28.707 . The traits with higher absolute value of characteristic vector were LL (0.935), PH (0.893), CD (0.884), PL (0.724), LC (0.737) and HS (-0.719). The numerical value of the second principal component was 3.432, and the contribution rate was 18.065 . The traits with higher absolute value of characteristic vector were SW (0.808), PW (0.791) and PT (0.694). The numerical value of the third principal component was 2.816 , and the contribution rate was $14.823 \%$. The traits with higher absolute value of characteristic vector were LM (-0.713), LT (0.668) and plant type (0.625). The numerical value of the fourth principal component was 1.737 , contribution rate was $9.141 \%$, and LW $(0.670)$ was higher. The numerical value of the fifth principal component was 1.112 , and the contribution rate was $5.852 \%$. The eigenvector value of RLN (0.677) was higher.

Table 5 Factor loading matrix and contribution rate of traits of test non-heading Chinese cabbage.

\begin{tabular}{|c|c|c|c|c|c|}
\hline \multirow[t]{2}{*}{ Traits } & \multicolumn{5}{|l|}{ Factor } \\
\hline & 1 & 2 & 3 & 4 & 5 \\
\hline $\mathrm{PH}$ & 0.893 & 0.001 & 0.020 & 0.177 & 0.085 \\
\hline $\mathrm{CD}$ & 0.884 & 0.076 & 0.098 & 0.187 & 0.123 \\
\hline RLN & -0.392 & -0.364 & -0.084 & -0.342 & 0.677 \\
\hline LL & 0.935 & -0.138 & -0.058 & 0.152 & 0.016 \\
\hline LW & 0.338 & 0.536 & 0.051 & 0.670 & 0.006 \\
\hline PL & 0.724 & -0.416 & 0.178 & 0.120 & 0.207 \\
\hline PW & 0.092 & 0.545 & 0.542 & -0.006 & 0.276 \\
\hline PT & -0.094 & 0.791 & 0.313 & 0.061 & 0.264 \\
\hline $\mathrm{SH}$ & -0.073 & 0.694 & 0.216 & -0.436 & 0.034 \\
\hline SW & 0.051 & 0.808 & 0.235 & -0.132 & 0.071 \\
\hline PT & 0.212 & -0.183 & 0.625 & -0.253 & -0.435 \\
\hline HS & -0.719 & 0.288 & -0.263 & 0.191 & -0.262 \\
\hline LT & -0.202 & -0.505 & 0.668 & 0.271 & 0.129 \\
\hline LS & 0.528 & 0.034 & -0.534 & -0.467 & 0.186 \\
\hline LTS & -0.587 & -0.112 & 0.467 & 0.443 & 0.104 \\
\hline $\mathrm{LM}$ & 0.249 & 0.390 & -0.713 & 0.194 & -0.143 \\
\hline LSS & -0.330 & -0.227 & -0.502 & 0.447 & 0.261 \\
\hline $\mathrm{LC}$ & -0.737 & -0.190 & 0.027 & -0.008 & -0.010 \\
\hline $\mathrm{PC}$ & -0.493 & 0.363 & -0.314 & 0.174 & 0.166 \\
\hline Numerical value & 5.454 & 3.432 & 2.816 & 1.737 & 1.112 \\
\hline Contribution rate & 28.707 & 18.065 & 14.823 & 9.141 & 5.852 \\
\hline Total account & 28.707 & 46.771 & 61.594 & 70.736 & 76.587 \\
\hline
\end{tabular}




\subsection{Cluster analysis of agronomic traits}

The 48 non-heading Chinese cabbage samples were analyzed by systematic clustering method, and the tested materials could be divided into 5 categories (Figure 1). The first group has one resource, which is Wuta-tsai. There were 16 resources in the second group, which were mainly Pak-choi with broad and light green petioles, small plant height and width, thick heads, green leaves and purple leaves. The leaves of code 2018332083 were purple. There were four resources in group III, all of which were erect-type leafy cabbages, with tall plants, large plant width, long leaves, long petioles and white petioles. There were 12 resources in group IV, which were mainly milk cabbage, with shorter plants, larger plant width, thick heads and wide leaves. There were 15 resources in group V, and the classification was mixed, mainly was erect-type leafy cabbage, but also 1 milk Chinese cabbage (P330226003) and 1 Pak-choi (P330127053). And some hard-to-divide materials formed by hybridization (P330127054, P330822020, P330502014) (Figure 2).

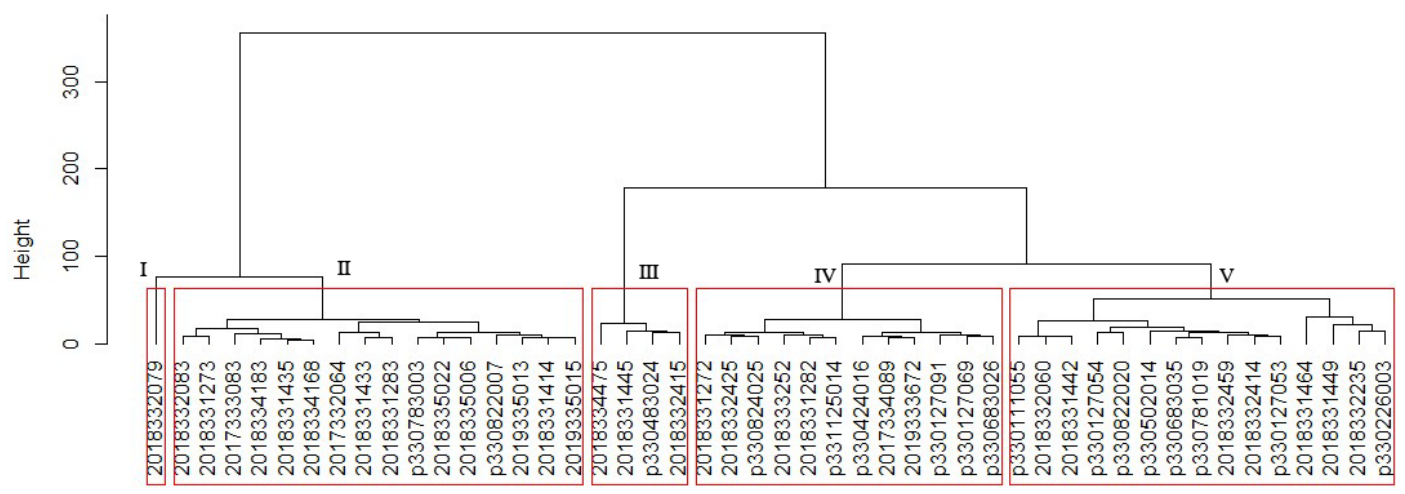

Figure 1 Clustering dendrogram of non-heading Chinese cabbage germplasm resources.

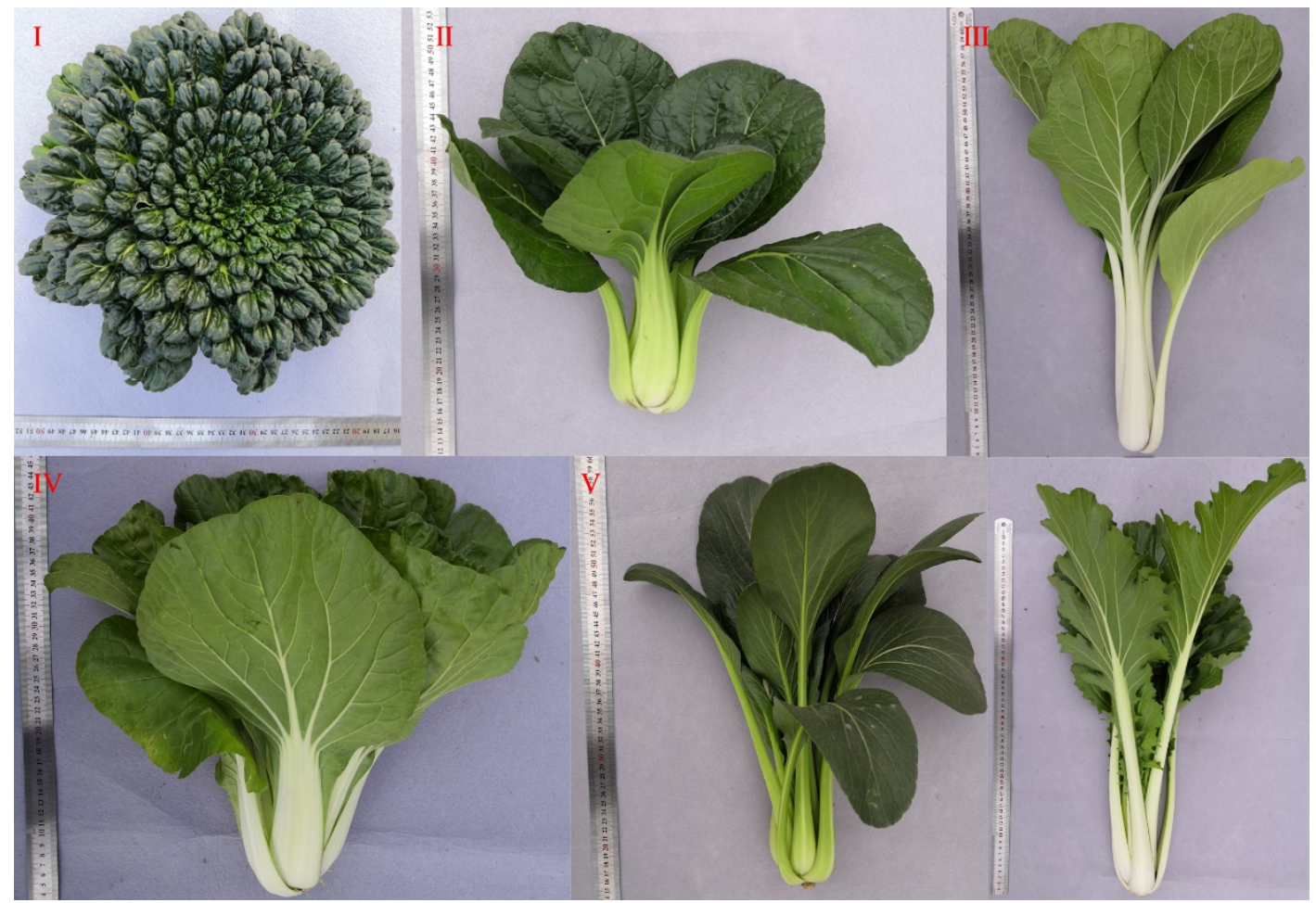

Figure 2 Representative plants of different types of non-heading Chinese cabbage germplasm resources

Note: type I: 2018332079; type II: P330783003; type III: P330483024; type IV: 2017334089; type V: P330822020; type V: P330127054, P330502014

\section{Discussion}

The genetic diversity of crop germplasm resources is evolved to adapt to the environment, and abundant variation is the genetic basis of variety selection. Analyzing the genetic diversity of crop germplasm resources can provide 
material basis for breeding. Rapid and simple identification of agronomic traits is the most basic and effective method to study germplasm resources (Xiang et al., 2018). Indicators such as coefficient of variation and genetic diversity index are commonly used to study population characteristic diversity (Han and Jiang, 2014). It has become a common practice to evaluate the diversity of crop germplasm resources based on phenotypic or genotypic data by principal component analysis, correlation analysis and cluster analysis, and has been widely used in genetic diversity research, classification and variety breeding of various crops (Nie et al., 2015; Wan et al., 2017).

The coefficient of variation reflects the degree of dispersion of characters. Previous studies believed that when the coefficient of variation was greater than $10 \%$, there was a large difference between samples for this trait (Lv et al., 2019). In this study, the variation coefficients of 19 phenotypic traits of 48 non-heading Chinese cabbage ranged from $14.57 \%$ to $65.77 \%$, all of which were higher than $10 \%$, indicating that there were abundant germplasm resources of non-heading Chinese cabbage in Zhejiang Province. Characters with coefficient of variation greater than $50 \%$ are petiole length, rosette leaves number, leaf margin and leaf shape. Petiole length showed high coefficient of variation in the studies of Sun (2014), Han (2010), Ma et al. (2015) and Xie et al. (2018). Leaf margin showed high coefficient of variation in the studies of Ma et al. (2015). Rosette leaves number and leaf type showed high coefficient of variation only in this study, which was not high in previous studies. However, there are also some traits with high coefficient of variation in previous studies, which are not particularly high in this study. These results indicated that the non-heading Chinese cabbage resources in Zhejiang had certain special characteristics, which could effectively broaden the genetic background of the non-heading Chinese cabbage population and provide material basis for its variety breeding.

The results showed that there were significant or extremely significant correlations among most of the characters of non-heading Chinese cabbage, indicating that different characters of non-heading Chinese cabbage were correlated and influenced each other. In this study, leaf length was significantly positively correlated with plant height, plant width and petiole length, and significantly negatively correlated with leaf number, which was basically consistent with the conclusion of Xie et al. (2018). However, Sun et al. (2014) believed that leaf length was significantly positively correlated not only with petiole length but also with leaf number, while Liu et al. (2014) believed that leaf length was not significantly correlated with leaf number. This may be related to the different test materials. Principal component analysis showed that the information content of the first five principal components accounted for $76.587 \%$ of the total information, which could effectively reflect the information content of many original trait variables. The first principal component included leaf length, plant height, plant width, petiole length, leaf color, hourglass sign and other traits, which were the target traits of breeding improvement. Cluster analysis could classify all materials according to phenotypic similarity. In this study, Wuta-tsai was a separate category, and Pak-choi, erect-type leafy cabbage and milk Chinese cabbage could also be clearly distinguished, but there was a hybrid category. In the previous clustering analysis of non-heading Chinese cabbage based on phenotypic data or molecular data, there were mixed groups (Han et al., 2010; Qin et al., 2013; Ma et al., 2015; Xie et al., 2018) showed high coefficient of variation, indicating high similarity of genetic background and phenotypic traits among different types of non-heading Chinese cabbage, with differences only in some key traits.

\section{Materials and Methods}

\subsection{Experimental material}

The test materials were non-heading Chinese cabbage collected from the Third National Survey and Collection Action on Crop Germplasm Resources in Zhejiang Province, which was collected and sorted by Vegetable Institute, Zhejiang Academy of Agricultural Sciences.

\subsection{Experiment design}

The experiment was carried out in Qiaosi Farm (Hangzhou), Zhejiang Academy of Agricultural Sciences. The materials were raised in mid-September 2019, transplanted in early October, harvested in late November, and routinely managed in the field. Phenotypic data were collected according to "Descriptors and Data Standard for Non-heading Chinese Cabbage". The investigated traits included plant height, crown diameter, rosette leaves 
number, leaf length, leaf width, petiole length, petiole width, petiole thick, stem height, stem width, per plant weight, plant type, hourglass sign, leaf type, leaf shape, leaf tip shape, leaf surface, leaf color, and petiole color. Three replicates were set for each material, and 5 plants were selected from each replicate for trait measurement.

\subsection{Data analysis}

The phenotypic data were collated by Microsoft Excel 2016. After statistics, the nine traits of non-heading Chinese cabbage, including plant type, hourglass sign, leaf type, leaf shape, leaf tip shape, leaf margin, leaf surface smoothness, leaf color and petiole color, were quantitatively processed according to "Descriptors and Data Standard for Non-heading Chinese Cabbage" (Table 6). Ten quantitative traits (PH, CD, RLN, LL, LW, PL, PW, PT, SH and $\mathrm{SW}$ ) were divided into 10 grades and assigned values. Grade 1 was defined as less than or equal to X-2 $\delta$, and grade 10 was defined as greater than or equal to $\mathrm{X}+2 \delta$, with a difference of $0.5 \delta$ ( $\mathrm{X}$ was the mean value and $\delta$ was the standard deviation). Shannon's diversity index was calculated using the relative frequency (Pi) of each level (He and Zong, 2011; Wang et al., 2014; Lv et al., 2020). SPSS 16.0 software was used for principal component and correlation analysis of traits, and R language program system clustering Ward method was used for cluster analysis.

Table 6 Classifying and assignment of quality traits

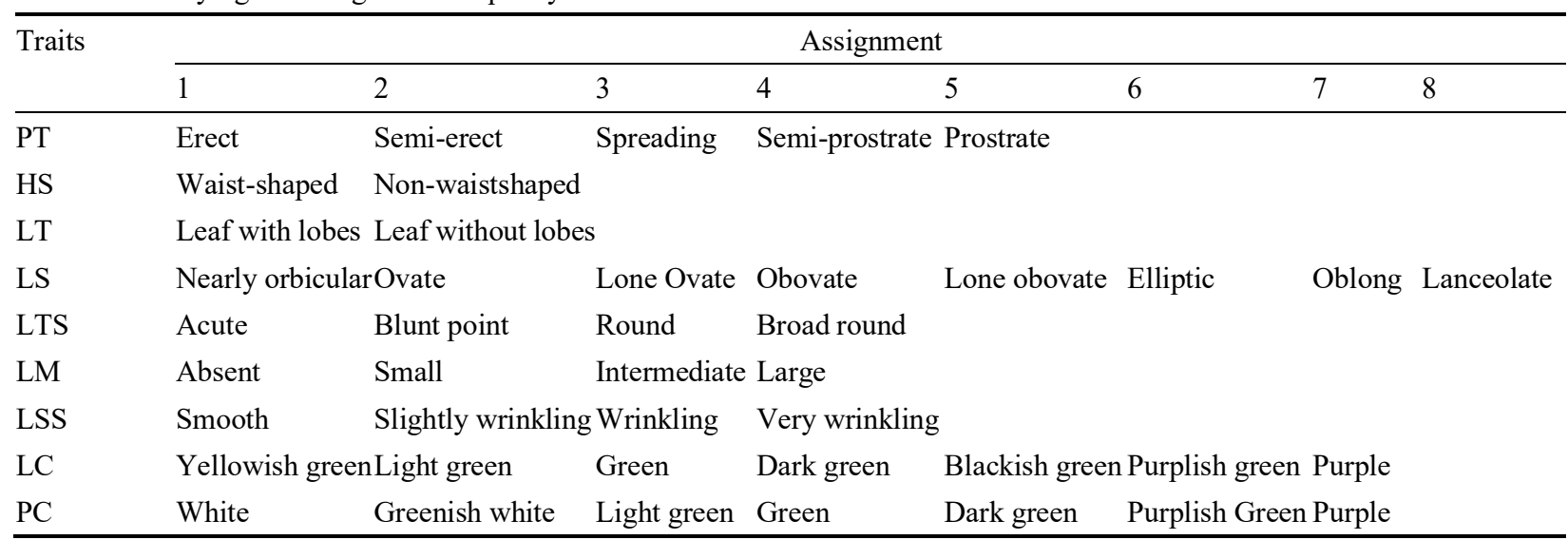

\section{Authors' contributions}

HHJ and WJL are the main executors of this study. HTH, WWH and WQZ were involved in data collection of phenotypic traits. BCL was the architect and principal of the project, directing experimental design, data analysis, paper writing and revision. All authors read and approved the final manuscript.

\section{Acknowledgement}

This study was supported by the Zhejiang Provincial Natural Science Foundation of China (LY19C060004), the Third National Survey and Collection Action on Crop Germplasm Resources in Zhejiang Province (111821301354052030), Zhejiang Science and Technology Plan Project-Research and Development and Demonstration of Horticultural Crop Varieties Based on Creative Agriculture (2018C02057) and Zhejiang Major Science and Technology Project-Breeding of New Varieties of Non-heading Chinese cabbage (2016C02051-6-3).

\section{References}

Feng X.H., Liu Q.Q., Zhou X.X. and Liu W.X., 2019, English translation of the names of various varieties of non-heading Chinese cabbage subspecies, Zhongguo Keji Fanyi (Chinese Science and Technology Translation Journal), 32(1): 34-35

Han J.M., Hou X.L., Xu H.M., and Shi G.J., 2010, Morphological diversity of non-heading Chinese cabbage (Brassica campestris ssp. chinensis Makino) germplasm, Shengwu Shuxue Xuebao (Journal of Biomathematics), 25(1): 137-146

Han Z.Q., and Jiang B., 2014, A study on comprehensive evaluation of the processing tomato varieties multiple traits, Zhongguo Nongye Kexue (Scientia Agriculture Sinica), 47(2): 357-365

He C.B., and Zong X.X., 2011, Genetic diversity of pea (Pisum sativum L.) germplasm resources revealed by morphological traits, Zhiwu Yichuan Ziyuan Xuebao (Journal of Plant Genetic Resources), 12(1): 42-48

Hou X.L., and Song X.M., 2012, Research and utilization of Brassica campestris ssp. chinensis Makino (non-heading Chinese cabbage), Nanjing Nongye Daxue Xuebao (Journal of Nanjing Agriculture University), 35(5): 35-42

Liu C.Y., Mu J.Y., and Sun L., 2014, The correlation analysis on main nutrient quality and agronomics traits of pakchoi, Beifang Yuanyi (Northern Horticulture), (15): $10-13$ 
Lv W., Han J.M., Ren G.X., Wen F., Wang R.P., and Liu W.P., 2019, Genetic diversity analysis of sesame germplasm resources in Shanxi, Zuowu Zazhi (Crops), 5: 57-63

Lv W., Han J.M., Wen F., Ren G.X., Wang R.P., and Liu W.P., 2020, Phenotypic diversity analysis of sesame germplasm resources, Zhiwu Yichuan Ziyuan Xuebao (Journal of Plant Genetic Resources), 21(1): 234-242

Ma J.J., Li N., Zhang S.J., Yu Z.J., and Hui M., 2015, Diversity analysis of non-heading Chinese cabbage based on seeding phynotypic traits, Beifang Yuanyi (Northern Horticulture), (17): 21-24

Nie S.H., Peng L., Wang X., and Ji L., 2015, Genetic diversity of agronomic traits in chickpea (Cicer Arietinum L.) germplasm resources, Zhiwu Yichuan Ziyuan Xuebao (Journal of Plant Genetic Resources), 16(1): 64-70

Qin Y.M., Li X., Zhao J.J., Wang Y.H., and Xuan S.X., 2013, Analysis of population structure of non-heading Chinese cabbage based on SSR marker, Huabei Nongxuebao (Acta Agriculturae Boreali-sinica), 28(3): 62-66

Sun L., Zhao F.L., Li Z.X., Wang G.Y., and Wu S.S., 2014, Genetic analysis and correlation analysis on agronomic traits of twenty-two Chinese cabbage, Beifang Yuanyi (Northern Horticulture), (17): 1-4

Wan S.W., Song F.J., Hao J.J., Zhang X.Y., Li H.W., Shao Y., and Zhao A.H., 2017, Genetic diversity of agronomic traits in 271 pea germplasm resources, Zhiwu Yichuan Ziyuan Xuebao (Journal of Plant Genetic Resources), 18(1): 10-18

Wang H.P., Li X.X., Shen D., Qiu Y., Song J.P., Zhang X.H., and Simon P.W., 2014, Evaluation on genetic diversity of garlic (Allium sativum L.) clones in china based on morphological characters, Zhiwu Yichuan Ziyuan Xuebao (Journal of Plant Genetic Resources), 15(1): 24-31

Wang Z.Z., 1988, Analysis on the development and utilization of gentle hill land in Zhejiang Province, Ziyuan Kaifa Yu Shichang (Resource Development and Market Journal), 4(2): 40-42

Xiang J.S., Xu F., Suo L.X., Cheng K., Wang Y.C., Meng H.L., Zhang J.L., Jia B., Wang D.X., and Diao X.M., 2018, Comparison on the phenotypic traits between landraces and cultivars of foxtail millet [Setaria italica (L.) P. Beauv.] in northeast China, Zhiwu Yichuan Ziyuan Xuebao (Journal of Plant Genetic Resources), 19(4): 642-656

Xie X.X., Zheng X.L., Lin F., and Shao G.R., 2018, Evaluation of 46 non-heading Chinese cabbage germplasm resources, (Acta Agriculturae Jiangxi), 30(2): 1620,25 